\title{
A Survey of MAC Protocols on Wireless Body Area Network
}

\author{
Khushbu Khalid ${ }^{1}$, Guo-Hui Li ${ }^{2}$ \\ ${ }^{1}$ School of computer science and Technology, Huazhong University of Science and Technology, Wuhan, China \\ ${ }^{2}$ School of Computer Science and Technology, Huazhong University of Science and Technology, Wuhan, China \\ ${ }^{1}$ khushbukhalid@yahoo.com, ${ }^{2}$ guohuili@hust.edu.cn
}

\begin{abstract}
Wireless sensor networks have traditionally been used for military purposes but now its usage has widened in different areas of life including medical terms which are called wireless body area network (WBAN). WBAN is playing a vital role in every aspect of medical science for monitoring the continuous health of patients' body without hindering the normal physiological activities in human body. All physiological data like a heartbeat, blood pressure and body temperature can be collected and then transferred to the data servers for further monitoring through WBAN. In this paper we will discuss about WBAN and some MAC protocols that has been designed to improve energy efficiency in WBAN
\end{abstract}

\section{Introduction}

Wireless sensor network is an ad hoc network in which a big amount of sensor nodes are connected to collect and deliver data. In the past, the researchers of wireless sensor network observed that this is not suitable for WBAN because WSN has a lot of sensors and all sensors are not working at the same time. It may cause data loss, more energy conservation and may deliver non-reliable data which does not give the accurate result. WSN depends on Bluetooth and 802.11 technologies, has been designed for the big data but for WBAN a small data delivery technology is needed because the radius of the human body is small and for big data delivery there may have a chance to lose the data. Therefore, it cannot deliver the emergency data and this cannot observe the heat of the human body. WAN is not directly attached to a network neither with a router nor with any other device, but the nodes helps to transmit the data. WSN is widely used in some areas like the channel of crowd and containers can be sorted, determine the impurities in ecology, examine the structure, recruit the surroundings, and control the traffic on roads and in health observations. As the health industry is progressing rapidly. Healthcare machines are establishing to monitor the health of human body and to improve the quality of life that's why researchers are more focused on health care devices to make them more reliable. WBAN is an application of WSN and it is designed for communication and to focus the physical parameters around the body like blood pressure, ECG, sugar level and heart rate etc. Generally, it is based on two devices sensors and actuators. The function of sensors is to observe the vital signs of the human body and the function of actuators is to take actions based on the data they receive from sensors. Basically, the sensors are attached to the internal or external body and continuously monitor health without disturbing the daily life and patients do not need to stay at the hospital. In WBAN, IEEE 804.15.4 protocol is designed in which data cannot be lost and emergency data can be delivered efficiently so that it can give more accurate results about human body temperature. As it is about the health of a human body so it is very sensitive if it may lose any of data. Therefore, we cannot compromise on this issue and more reliable health care devices are used. The major problems are the life of the battery which is installed in a body. Moreover, the battery is nonrechargeable and cannot even replaceable which are the drawbacks in WBAN. Previously, many protocols had been designed in WSNs but that protocols are unable to fulfil the requirements of WBANs because it might work in the different environment and also the design is quite different. Generally, redundancy occurs in WSN because a big amount of sensor nodes are densely attached to gather the data and sends it to all the nodes and all nodes cannot work at the same time but in WBAN only 256 nodes are attached to the human body. Data is sensitive in WBAN as all nodes are active and data is more reliable to transfer as compared to WSN. It is the cause of energy consumption because all the nodes are working at the same time so in health measuring process of the human body so there is a need for protocols and techniques that may consume less energy in order to observe the health continuously. The whole communication system of WBAN can be categorized in three tiers. In tier1 the sensors which are installed inside the human body communicate to each other and this communication can be termed as intra-body communication. In tier2 the sensors which are implemented on the human body communicate with the 
personal device. This communication can be termed as extra-body communication. Tier3 is also an extra-body communication where personal devices transfer the collected data to the server.

\section{Mac Protocol Design and Challenges}

Lifetime of a network is the main objective of WSN. In this portion. we have explained about the energy efficient MAC protocols with some sources of energy wastes which tells the occurrence of energy wastes in the network and basic properties of a stable network

\subsection{Attributes of a Good Protocol}

Some of the attributes that are required to design a good MAC protocol are discussed below.

Energy Efficiency: Energy efficiency is an important attribute in the protocols. It is better to change the sensor node than to recharge the battery because the energy is being used either to send the data or it is kept on for a long time. MAC is proposed to monitor the energy while it is working or not working. It should help to stop the energy wastes. It has to sleep or turn off mode when nothing is transferring. Sometimes WBAN has to monitor the patients for a long time so MAC protocol should have to work efficiently and look after all the usage of energy in the network in order to save energy like from retransmission or collision. MAC protocol should help sensor node to turn off or go to sleep mode to decrease the waste of energy and can operate at low power.

Latency: It is required to observe the events in all nodes and sends the information to the base station (sink) and it can take the actions immediately to avoid all the collisions in the future. It may also relate to the delivery of packets because it's the time or the duration in which the packets are reaching the destination. In some applications there is a chance to the occurrence of short latency and short latency can be fatal in case of WBAN. The packets should be delivered on time even if the medium is not reachable it can use intermediate nodes to send packets to reach on time and to avoid worst scenarios.

Throughput: It is data transfer from node to the destination per unit time. It can be used in different applications in different ways. Some of the reasons like packets collisions, retransmission and latency, can be responsible for effecting the throughput. It also depends on patient's parameter because some patients need to be monitored regularly and some monitored irregularly.

Mobility: One of the most likeable attributes is mobility because if the patient needs to be monitored continuously at the hospital he can feel uncomfortable. No one is happy to stay at the hospital for a long time. Patient is allowed to go anywhere and no need to stay at the hospital and the health of the patient can be measured without disturbing the daily life.

Fairness: It is compulsory for the sensor networks that the packets receiving by the nodes are reaching properly to the sink. Although it depends on the energy efficiently and throughput, so energy efficiently increased by energy wastes. One node may have more data send with others so it needs to cooperate with all and reach on time to the destination.

\subsection{Major Sources of Energy Wastes}

Collision: During the process of transfer of data, some of the packets are being corrupted because of the interference and the nodes try to send the data again due to which it consumes a lot of energy. It is obvious that receiver has to choose any of the packets and drop the packet if they reach at the same time. As a result, it requires more energy in order to receive other packets that are delayed.

Overhearing: During the heavy traffic, overhearing is not good because it can causes major issues like if the packets are supposed to reach the given destination but they reach to another place.

Control packet overhead: When a small byte of data is transferred to the node it usually consumes more energy that is unacceptable for the network to use an energy that is responsible just for sending a small size data which decrease the capacity of the channel. To control this process it requires a fine approach that handles the situation.

Over emitting: It may happen in some cases that the receiver is not ready to receive the packets. Sometimes the traffic is so busy that receiver cannot able to handle all the packets at the same time and because of this error, there is greater chance to lose the packets.

Idle listening: Most of the time the sensor nodes are in idle state because if no data is transferring it may also listen to the network which is one of the big causes of energy wastes

\section{Energy Reduction Methods In Mac Protocols for Wban}

As the energy of the battery cannot be used for a lifetime as sometimes the patients need monitoring without any interruption. To save the battery usage it can be required in three places, sensing the data, data processing and communication. During the communication, a big amount of energy is used because idle listening, over emitting and traffic consumes the battery. To stop this consumption duty cycling is applied, it can increase the network life because it controls the idle listening. Sleep and active mode increase the performance of the network and saves its energy. There are three methods to save energy, low power listening LPL, schedule contention and TDMA.

\subsection{Low Power Listening}

In this technique sleep and wake up mode is determined all nodes are sleeping and just one node is awake to see the channel if packets are coming or not. It is also known as channel pooling. Because of this sleep and wake-up technique, most of the energy is saved. The nodes can only wake up at the time of receiving packets. LPL works well among networks because it has fixed data rate and it's very sensitive to the data variation. Its performance is tainted in the state of highly changing data traffic rates. However, it increases efficiently for already known periodic traffic rates. Wise-MAC is an LPL based MAC protocol. This 
protocol reduces idle listening by employing non-persistent CSMA and preamble sampling techniqu

\subsection{Schedule Contention}

For WBANs contention based MAC protocol is not good because it is very sensitive to lose the data. Schedule based MAC, all the contenders (sensor nodes) contend for the channel before transmitting data. This mechanism is not acceptable for health issues because it has a chance to the collision of data packets and loss of packets. To stop and avoid collision both scheduled and contention based MAC are used together. Nodes are allowed to follow a specific schedule for communication of data. It is also a cause of extra energy because of different clusters. It can schedule differently. As MAC is a multi-hop network in WSN. By default, the duty cycle is applied to all nodes which are the basic function of this technique. Some of the MAC protocols based on scheduling are, TDMA, CDMA and FDMA. High computational overhead and limitation of sensor nodes, CDMA and FDMA are not recommended to use in WBANs. The nodes are only active while sending data otherwise keep sleeping. When data packets and synch reach simultaneously the node is dividing in two parts. The frame is the complete cycle of listening and sleep and cycle starts from the wake-up mode. If the node is sleeping it wait until it wakes up.

\subsection{TDMA}

In this mechanism, a base station gives time slots to all nodes. TDMA based MAC protocols performance basis on the traffic amount of the network. TDMA based Macs are efficient in the scenarios when networks have more traffic because the channel is efficiently used. If network traffic isn't enough and sensor node doesn't have data to send or receive then all other nodes need to wait for their slots and it causes wastage of the bandwidth. TDMA is the best mechanism because it increases power efficiency, just because it wakes up only at the time of transmission otherwise it sleeps all the time and it's also best for the synchronization of devices.

\section{Energy Efficient MAC Protocol}

WSN is growing so fast and the sensor nodes consume a lot of energy. In order to save the energy researchers have designed some electronic devices that consume less energy. By using these devices some protocols of energy efficiency have been designed. (MAC) medium access control is the technique that basically used to avoid the collisions from interfering nodes. The function of protocols is to define a mechanism that communicates each other in such a way that able to control the traffic and gives the reliability so the packets reach the destination. It also helps to organize nodes and sequence of the network in such a way that the sender and receiver communicate each other without collision. There are two types of channel Contention based channel and Contention free channel .Before the transmission of data, it does not allocate the channel to sensor nodes. Every node is struggling for the channel is contention based. If the nodes sends the data and at the same time the channel is busy then the node will wait until the channel is free and then sends the data to the channel and the state of sensing channel is called clear channel assessment (CSA) and in contention free mechanism which is TDMA, every node has given a specific time slots in which it has to send the data and the frame is organized by base station which is also called central node (sink) and the node has to follow the organization blindly. Similarly the sensor nodes never transfer the data to the node to the node which is not allocated to it.

\subsection{Body Mac}

To reduce energy TDMA based MAC protocol was proposed. In this protocol uplink and down link were defined and nods will be in sleeping unless they have no data. It is also good in low duty cycle. Burst bandwidth procedure, periodic bandwidth procedure and adjust bandwidth procedure are three types of bandwidth procedures. The bandwidths that have periods and reduces bandwidth to the half is burst bandwidth, in periodic bandwidth each part of frames in MAC and stored and every part has the special access to the channel. Total bandwidth that needs to increase or to decrease from periodic bandwidth is adjusting bandwidth. Some of the drawbacks are that the node can sense the data and transfer it even if it is in sleep mode, so in an emergency it is highly useful protocol to send the data. The first one is a beacon which is responsible for synchronization of data. The second part is downlink which is used to manage the process of transferring the data from the coordinator. The last one is uplink which is further divided into two parts contention access period (CAP) and contention free period (CFP).in CAP nodes fight to send the packets and responsible for sending small packets. CFP is basically used to increase the communication effecting energy

\subsection{Med Mac}

To improve the channel access mechanism Med Mac was designed. It is also used for reduction of energy. To communicate the data the slots they have given are the usually different size and different lengths. This protocol is used to initialize the network or for traffic emergency. Sometimes it is used for communication of low data rates, time stamp scavenging with adaptive guard band algorithm (AGBA) used in med Mac.

Generally, a guard time is used between two consecutive time slots using AGBA, this guard time can be adjusted and depends upon clock drifts. Drift adjustment factor (DAF) is used to guard band and helps to stop the extra guard bands. With a comparison with IEEE 802.15.4, it is used for low data rates like temperature, respiration and pulse etc. and medium data rates like ECG. For WBAN it is not suitable for high data rates and Med Mac can be used only for low and medium data rates.

\subsection{Energy Efficient Low Duty Cycle}

This is a new protocol with a static nature. This protocol is used for a large amount of data; the static nature of this protocol increased the life of the network. The master node (MN) is used to gather data from nodes and transmits by 
the monitoring station. MS is used to observe the receive data which is also responsible for synchronization. This protocol has the static nature with fixed data rate generation. It is not suitable for the dynamic type of network. It is TDMA base protocol. Guard time (Tg) is used between each time slots. This Tg helps to avoid collisions between time slots .this is the protocol that decreases the idle listening. It is widely used in the medical area. For example, to monitor patients no need for the assistant. Minimum power is used. To monitor ECG it can be used which is very important in the field of medicine

\section{D. IEEE.802.15.4}

It builds a connection among all the devices and it can consume less power, low cost and data rates are also low.IEEE.802.15.4 consists of three parts that are used in super frames. ADAPTED CSMA/CA, ALGORITHM and GUARANTEED TIME SLOTS. Contention access period (CAP) and contention free period (CFP) are used for these schemes. Generally, IEEE.802.15.4 has two protocols that are random and scheduled. Traffic latency is very low is CSMA /CA. Star topology is used in WBAN and TDMA mechanism is being required in MAC protocols in WBAN. If it is compared IEEE.802.15.4 protocol it has used the concept of master/slave communication.IEEE.802.15.4 is not suitable for BANs platform because it has low data rate, low flexibility and power consumption. Some of the protocols are defined below to examine the energy consumption. IEEE 802.15.4 has the ZigBee technology and has two types of devices.

FFD (full function device): It is usually the router the router or gateway or coordinator of the network which helps with other networks.

RFD(reduced function device): It has low power consumption and low complexity.

IEEE.802.15.4 is divided into three bands of frequency $868 \mathrm{MHz}, 915 \mathrm{MHz}$ and $2.4 \mathrm{GHz}$ and they are divided into 27 sub channels. $2.4 \mathrm{GHz}$ has 16 sub channels, $915 \mathrm{MHz}$ has 9 sub channels, and $868 \mathrm{MHz}$ has 1 sub channel. It consists of two operational modes. Beacon enabled mode and non-beacon enabled mode. Beacon enables wises a super frame that has active and inactive parts. Active period has three parts which are contention access period, beacon and contention-free period.To support critical data end node are assigned seven guaranteed time slots but because of this kind of transmission this is not suitable for WBAN and CSMA/CA is used in non-beacon mode.

\subsection{E. Enhanced MAC Protocol}

The concept of IEEE 802.15.4 has been extended by CHEOLLYO LEE at el and it is clear that this protocol is not suitable for all types of applications. It is useful only for the devices that are used for health care. To measure human body a WBAN does not need many more devices but it needs a connection to some hops. IEEE 802.15.4 is not supportive to send the emergency data because in medicine there are some moments in which data needs to send in an emergency. Contention based medium is also known as CSMA/CA and guarantee time slots GTS both methods are used by the frame of the IEEE.802.15.4. This protocol cannot fulfil the requirements of WBAN because for WBAN critical time is the basic requirement and data needs to send emergency. This protocol is required to send the data quickly. Beacon is responsible for the periodic synchronization among BAN coordinator and other nodes. Super frame consists of primary beacon (PB), polling period (PB), emergency slot (ES), secondary beacon (SB), contention access protocol (CAP) and contention fee period (CFP). At the end of super frame an active period can be added .polling period and emergency time slot can be used for the extension of the function of the super frame with CAP and CFP. The devices that have installed inside or outside the body used only two-hop communication. Moreover, the division of slot interval is big and proficiency is low.

\subsection{F.Extended Enhanced MAC Protocol}

It has been extended by this algorithm which is based on IEEE.802.15.4 concepts. Super frame of IEEE.802.15.4 is given below. Contention based (contention access period) and contention free access mechanism (guaranteed slot time) both techniques has been supported by this algorithm

It supports both, the contention based (contention access period) and contention free access mechanism (guaranteed time slot).It does not provide any solution for the emergency data and for guarantee delivery of date. On emergency data, sensor nodes need to send the data. It cannot wait for the time slot. Therefore, to transfer the data fast emergency slot (ES) has been used with the contention access period (CAP), contention free period (CFP), polling period $(\mathrm{PP})$, primary beacon $(\mathrm{PB})$ and secondary beacon (SB).

In the super frame structure of Enhanced MAC protocol. Along with ES, Contention Access Period (CAP), Contention Free Period (CFP), Polling Period (PP), Primary Beacon (PB) and Secondary Beacon (SB) have also been used.

Primary beacon (PB) is responsible for the BAN coordinator and the devices to synchronize. The basic purpose of PB is to be with super frame information e.g. beacon order, super frame order and slot insights excluding the CFP specifications. In PP the node that waits for polling request is basically responsible for the extraction of data. ES is used for transmission of emergency data. ES has also very short interval because the emergency event occurs rarely. IEEE 802.15.4 and Enhanced MAC protocol generally support one or two hops communication. As the nodes are implanted on both sides of the body the back side as well as the front side and because of the shadow of the body there is a chance of losing the packet. BAN coordinator is responsible for sending the data in Enhanced MAC protocol but all the nodes unable to get the information due to its two-hop connectivity. For the enlarge delivery of packets ratio EEM (Extended Enhanced protocol MAC) proposed a tree-based hierarchy which helps to keep remain the connection up to multiple hops. In EEM, the primary beacon has been send to all nodes by BAN coordinator. First hop and BAN coordinator participate in primary period. The emergency slot has been used for the emergency data and the CSMA/CA technique has been used which is started by the 
nodes at first hop and BAN coordinator in order to deliver the packets from nodes. Then secondary beacon has been sent by the personal server to all nodes. In CFP contention free period, the nodes are multi-hops and they require to send packets from fixed slots because of no direct connection with P.S, the nodes can transmit packets at first level of hierarchy but failed on other levels, so Tree Based Hierarchy has their super frame which allows other nodes to work as coordinator at multi-hop nodes in guaranteed time slot (GTS).

\section{Conclusions}

In this paper we discuss about the WSN and its charactristics along with different energy efficient MAC protocols. In BSN, Body sensor networks reliability of long life of the battery is the necessity that can be supported on the patient's body. The concept of developing energy efficient MAC protocol has become popular and exciting and it has been a good area of research over last decade. Reliability and energy efficiency are the fundamental issues that should take care of.

We also explained the extended enhanced MAC protocol in which tree-based hierarchy has been used which is not limited to only two-hop communications and I observe that it increases the reliability as compared to other previous protocols.

More work can be done for the improvisation of average and maximum delay of extended enhanced MAC protocol in the future.

\section{References}

[1] Giuseppe Anastasi, Marco Conti, Mario Di Francesso, et al. Energy conservation in wireless sensor network: A survey, Adhoc Networks, 2008, 20(7):537-568.

[2] S.R. Gandham, M. Dawande, R. Prakash, et al. Energy efficient schemes for wireless sensor networks with multiple mobile base stations, in: Proc. IEEE Globecom 2003, San Francisco, CA December 1-5, vol. 1, 2003, pp. 377-381.

[3] V. Raghunathan, C. Schurghers, S. Park, M. et al. Energy-aware wireless microsensor networks, IEEE Signal Processing Magazine (2002) 40-50.

[4] G. Anastasi, M. Conti, M. Di Francesco, et al. How to prolong the lifetime of wireless sensor networks: 2004, pp.234-236.

[5] M.C. Vuran, O.B. Akan, I.F. Akyildiz, Spatiotemporal correlation: theory and applications for wireless sensor networks, Computer NetworksJournal 45 (3) (2004) 245-261.

[6] E. Fasolo, M. Rossi, J. Widmer, et al. In-network aggregation techniques for wireless sensor networks: a survey, IEEE Wireless Communications 14 (2) (2007).

[7] S. Ullah, P. Khan, N. Ullah, S. Saleem, et al. Areview of wireless body area networks for medical applications, vol. abs/1001.0831, 2010.
[8] D. Smith, D. Miniutti, T. A. Lamahewa, and L. Hanlen, Propagationmodels for body area networks: A survey, IEEE Antennas and Propagation Mag., Dec. 2013.

[9] J. Y. Khan, M. R. Yuce, G. Bulger, and B. Harding, Wireless bodyarea network (WBAN) design techniques and performance evaluation,J. of medical systems, 2012, vol. 36, no. 3, pp. 1441-1457.

[10]A. Zhang, D. Smith, D. Miniutti, et al.Performance of Pico-net co-existence schemes in wireless body areanetworks,IEEE Wireless Commun. andNetw. Conf. (WCNC), April 2010. pp. 1-6.

[11]R. Shah and M. Yarvis, Characteristics of on-body 802.15.4 networks,2nd IEEE Workshop on Wireless Mesh Networks (WiMesh), Sept. 2006,pp. 138-139.

[12]E. Reusens, W. Joseph, B. Latre, et al. Characterization of onbody communication channel and energy efficient topology designfor wireless body area networks,IEEE Trans. Inf. Technol. Biomed.,Nov. 2009, vol. 13, pp. 933 -945.

[13]Polastre J, Hill J, Culler D, Versatile low power media access for wireless sensor networks. Proceedings of International Conference on Embedded Networked Sensor Systems; NewYork, NY, USA. 2004, pp. 95-107.

[14] Wei Y, Heidemann J, Estrin D, An energy-efficient MAC protocol for wireless sensor networks, Proceedings of IEEE Infocom 2002; New York, pp. 1567-1576.

[15] Van Dam T, Langendoen K, An adaptive energy efficient MAC protocol for WSNs. Proceedings of ACM Conf on Embedded Networked Sensor Systems, SenSys 2003, pp. 171-180.

[16]Zhen B, Li HB, Kohno R, IEEE body area networks for medical applications. Proceedings of IEEE International Symposium on Wireless Communication Systems 2007.

[17] Cheolhyo Lee, Hyung-Soo Lee, et al. An enhanced MAC protocol of IEEE 802.15.4 for Wireless Body Area Networks, Dec 2010, pp. 916-919.

[18]A. Milenkovic, C. Otto, et al. Wireless sensor networks forpersonal health monitoring: Issues and an implementation,ComputerCommunications, 2006 vol. 29, pp. 2521-2533.

[19] C. Otto, A. Milenkovic, et al. System architecture of a wireless body area sensor network for ubiquitous healthmonitoring,J. Mob. Multimed, Jan. 2005, vol. 1, pp. 307-326.

[20]D. Smith and L. Hanlen, Wireless body area networks: Towards awearable intranet, ISCIT Tutorial, Sept. 2012.

[21]M. Chen, S. Gonzalez, et al. Bodyarea networks: A survey, Mobile Networks and Applications, 2011, vol. 16,pp. 171-193.

[22] S. Ullah, B. Shen, et al.A study of medium access control protocols for wireless body areanetworks,arXiv preprint arXiv:1004.3890, 2010. 
[23]H. Kwon and S. Lee, Energy-efficient multi-hop transmission in bodyarea networks,20th IEEE Int. Symp. on Personal, Indoor andMobile Radio Comm. (PIMRC), Sept. 2009, pp. $2142-2146$.
[24]B. Latr'e, B. Braem, et al. Asurvey on wireless body area networks, Wirless Network, Jan. 2011, vol. 17,pp. $1-18$.

[25]I.F. Akyildiz, W.Su,et al. Wireless sensor networks: A survey, Computer Networks 38(4)(2002) 